\title{
Tratamiento quirúrgico de la endocarditis infecciosa en un hospital general: Indicaciones y morbi- mortalidad
}

\author{
Surgical treatment of infectious endocarditis in a general hospital: Indications for and morbi-mortality \\ Carla Saito ${ }^{1, a}$, Miguel Padilla ${ }^{1, a}$, Andrea Valle ${ }^{1, a}$, Enrique Castañeda ${ }^{1,2, b, c}$ \\ RESUMEN
}

Objetivos: Describir las indicaciones de tratamiento quirúrgico de la endocarditis infecciosa, el momento de indicación quirúrgica, tipo de cirugía y morbi-mortalidad post-operatoria. Material y métodos: Estudio descriptivo y retrospectivo. Se incluyeron pacientes con diagnóstico de Endocarditis Infecciosa (EI) definido según los Criterios de Duke, que no respondieron al tratamiento médico y que además contaban con indicación de tratamiento quirúrgico de acuerdo a la evaluación de los especialistas del Servicio de Cirugía Cardiovascular del Hospital Nacional Cayetano Heredia, desde enero del 2000 a diciembre de 2013. Se revisaron las historias clínicas y se determinaron las características clínicas de la población, indicaciones quirúrgicas, momento y tipo de cirugía, mortalidad y complicaciones. Resultados: De 27 pacientes con diagnóstico de EI e indicación de tratamiento quirúrgico, el $74,07 \%$ tuvo indicación de cirugía por riesgo de embolismo, el 70,37\% por insuficiencia cardiaca refractaria a tratamiento médico y el 51,85\% por infección no controlada. El 77,78\% tuvo indicación de cirugía de urgencia y el 22,22\% indicación de cirugía de emergencia. El 25,93\% de los pacientes fue intervenido quirúrgicamente. A 6 pacientes se les realizó cambio valvular. Un paciente falleció luego de la cirugía y otros 3 presentaron complicaciones post-quirúrgicas. Conclusiones: La indicación más frecuente fue el riesgo de embolismo indicado como cirugía de urgencia. La válvula mitral fue la más afectada y la mayoría fue sometida a cirugía de cambio valvular. La mortalidad post-operatoria fue $14,28 \%$ y las complicaciones post-operatorias frecuentes fueron sangrado torácico y coagulopatía.

PALABRAS CLAVE: Endocarditis bacteriana, endocarditis, defectos de la almohadilla endocárdica. (Fuente: DeCS BIREME).

\section{SUMMARY}

Objectives: To describe the indications for surgical management of infective endocarditis (IE), timing of surgical intervention, type of surgery and post-operative morbi-mortality. Methods: Retrospective study that enrolled patients with IE following Duke's criteria that did not respond to medical treatment, with indications for surgical management based on an evaluation by cardiothoracic specialists of Hospital Nacional Cayetano Heredia from January 2008 to December 2013. Clinical charts were reviewed in search for indications for surgical management of IE, timing of surgical intervention, type of surgery and post-operative morbi-mortality. Results: A total of 27 patients were diagnosed with IE during the study period; $40.7 \%$ had indications for surgical intervention due to risk of embolization; $70.37 \%$ for heart failure not responding to medical treatment and $51.85 \%$ for uncontrolled infection; $77.78 \%$ had indication for urgent intervention and $22.2 \%$ of emergency intervention. A total of $25.93 \%$

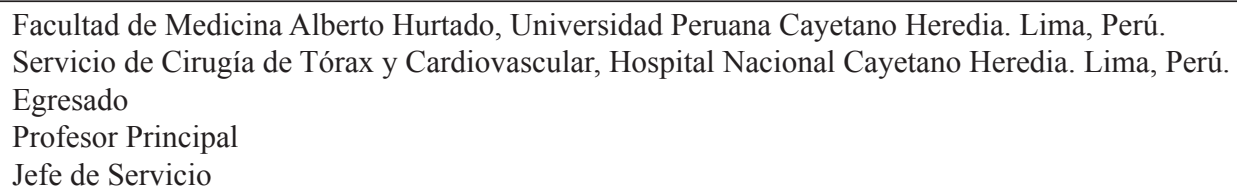


were surgically intervened; 6 patients underwent valve exchange. One patient died after surgery and 3-presented post-surgical complications. Conclusions: The most frequent reason for surgical intervention was risk of embolism, the mitral valve was the most affected and valve exchange was the most common procedure performed. Postoperative mortality was $14.28 \%$, post-surgical bleeding and coagulopathy were the most frequent post-operative complications.

KEYWORDS: Endocarditis; endocarditis, bacterial; endocardial cushion defect. (Source: MeSH NLM).

\section{INTRODUCCIÓN}

Durante los últimos años se han desarrollado múltiples estudios acerca del diagnóstico y manejo de la endocarditis infecciosa. A pesar de los avances registrados, sobretodo en cuanto al diagnóstico temprano de ésta enfermedad, la mortalidad sigue permaneciendo elevada llegando a alcanzar hasta el $50 \%$ en algunos estudios (1).

El tratamiento óptimo de la endocarditis infecciosa se basa en una combinación de tratamiento antibiótico adecuado y prolongado, y escisión quirúrgica de las estructuras afectadas, pudiendo llegar a ser necesario hasta en 40 a $50 \%$ de los pacientes (2).

En diversos estudios se ha demostrado que, en ciertas indicaciones, el manejo quirúrgico unido al adecuado tratamiento antibiótico ha resultado beneficioso, disminuyendo la incidencia de secuelas y complicaciones, así como la mortalidad. Sin embargo, el tipo y el momento de realización de la cirugía aún permanecen en debate y muchas veces, quedan a decisión del cirujano según su experiencia y la situación particular de cada paciente $(1,3,4)$.

Debido a esto, y a pesar de la existencia de guías internacionales, el manejo de éstos pacientes no es uniforme, representando un gran problema, sobretodo en nuestro medio, donde aún existen pocos estudios relacionados al tema (5).

El estudio tuvo como objetivo describir las indicaciones de manejo médico y quirúrgico de los pacientes con endocarditis infecciosa en un hospital general, enfatizando las indicaciones quirúrgicas, tipo y momento de la cirugía y morb-mortalidad postoperatoria

\section{MATERIAL Y MÉTODOS}

Estudio retrospectivo y descriptivo, realizado en pacientes con diagnóstico de endocarditis infecciosa (EI) hospitalizados en el Hospital Nacional Cayetano Heredia (Establecimiento de Salud nivel III-1 y hospital de referencia de alta complejidad) entre enero del 2000 y diciembre de 2013. Se incluyeron en el estudio los pacientes con diagnóstico de EI definido según los criterios de Duke (6) que no respondieron al tratamiento médico y que tenían indicación de tratamiento quirúrgico de acuerdo a la evaluación de los especialistas del Servicio de Cirugía de Tórax y Cardiovascular. No se excluyeron pacientes.

Se solicitó a la Oficina de Estadística de dicho Hospital las historias clínicas de los pacientes con diagnóstico de egreso de EI, mediante los códigos CIE10 I33.9, I38 e I39, correspondientes a endocarditis aguda o subaguda, endocarditis de válvula no especificada y endocarditis y trastornos valvulares en enfermedades clasificadas en otra parte.

Se identificaron 106 historias clínicas, 37 no se encontraron en el archivo o estaban incompletas, 13 no tuvieron diagnóstico de EI según los criterios de Duke modificados, cinco no contaban con indicación de tratamiento quirúrgico por el Servicio de Tórax y Cardiovascular y 24 respondieron favorablemente al tratamiento médico durante su evolución; finalmente, se incluyeron en el estudio 27 pacientes (Gráfico 1).

Los datos fueron analizados utilizando el programa estadístico STATA/MP versión 12.0 y MS Excel versión 2010. Se utilizó estadística descriptiva determinando frecuencias y porcentajes.

El estudio fue revisado y aprobado por el Comité de Ética de la Universidad Peruana Cayetano Heredia.

\section{RESULTADOS}

La relación hombre/mujer fue 3,5:1, la media de edad fue $42,11 \pm 18,82$ años. Dentro de los factores predisponentes, los más frecuentes fueron procedimiento dental e inmunosupresión (Gráfico 2).

En 25 pacientes se tomó muestra de hemocultivo, fueron positivos en $12 \quad(48 \%)$, aislándose Staphylococcus sp. en 5 (41,67\%) pacientes, Streptococcus sp. en 3 (25\%) y Enterococcus sp. en 2 


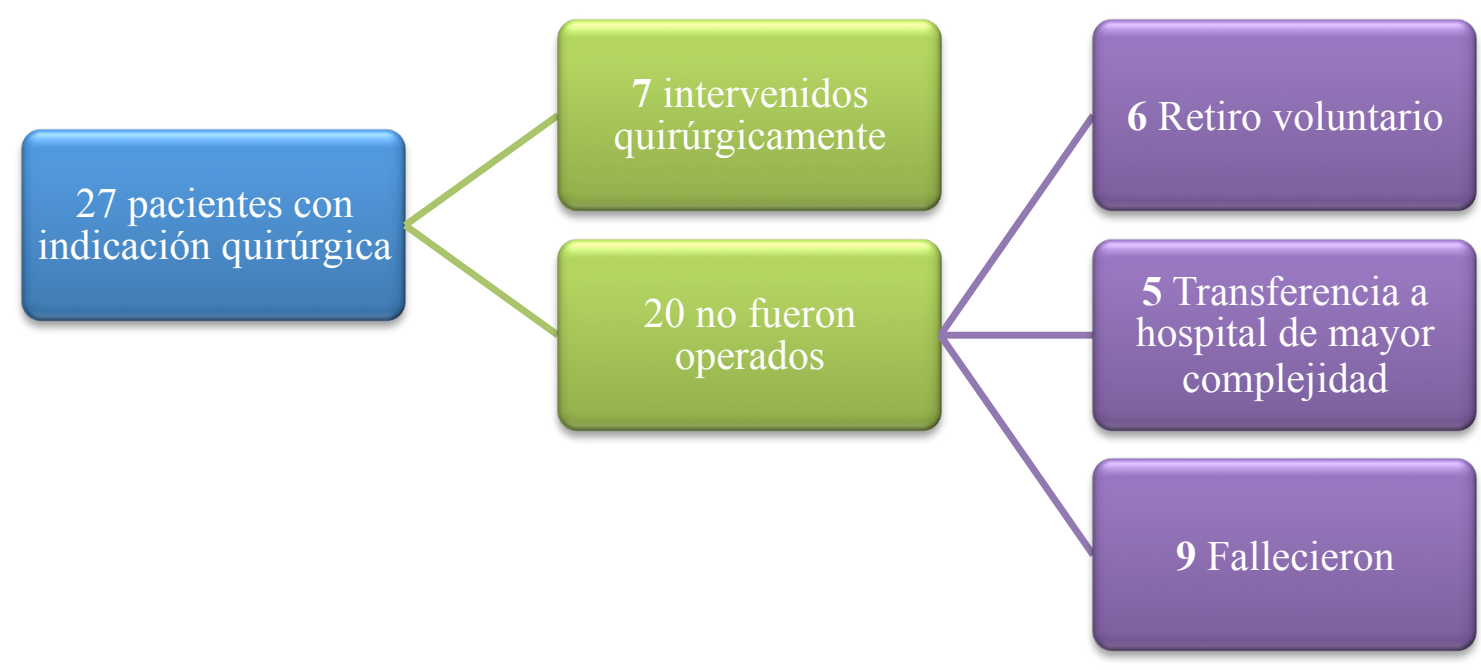

Gráfico 1. Flujograma de pacientes con indicación quirúrgica.

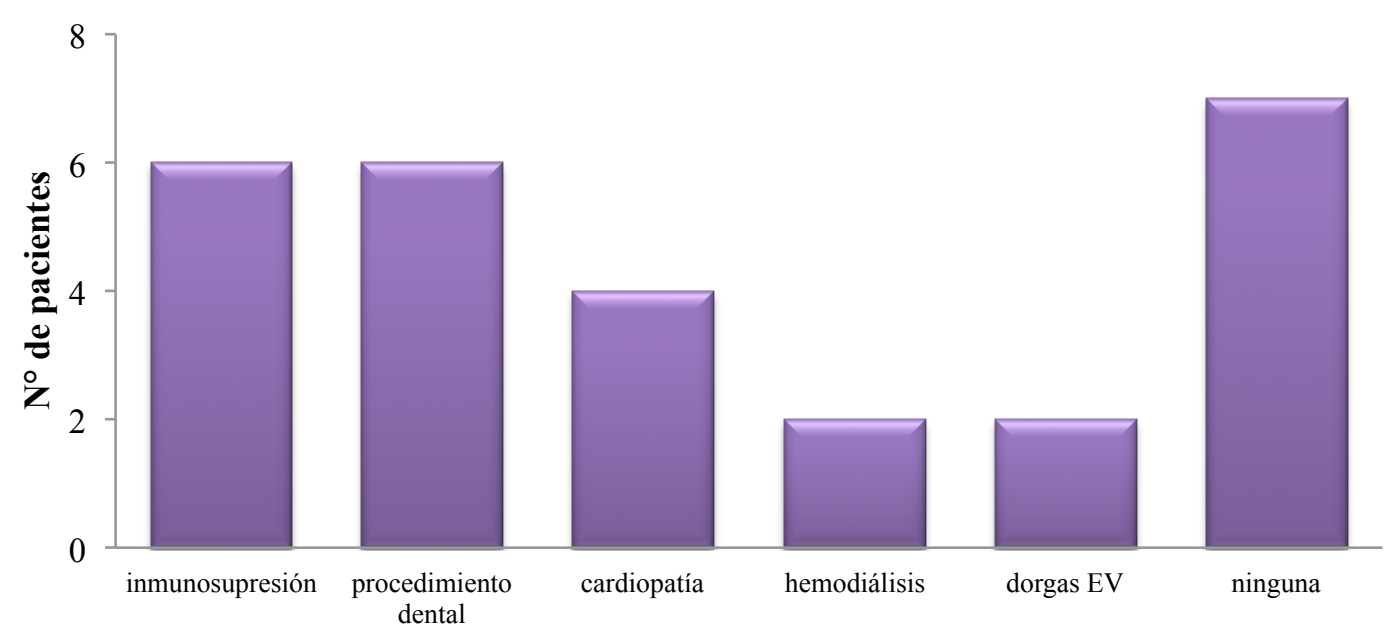

Factores predisponentes

Gráfico 2. Factores predisponentes de Endocarditis Infecciosas.

(16,67\%). Los antibióticos más comúnmente usados fueron Gentamicina en $21(77,78 \%)$, Vancomicina $16(59,26 \%)$, Ceftriaxona $12(44,44 \%)$ y Penicilina $\mathrm{G}$ en $11(40,74 \%)$. Ningún paciente recibió profilaxis antibiótica.

Las válvulas afectadas con más frecuencia fueron la mitral y la aórtica (Tabla 1). En 7 (25,9\%) se afectó más de una válvula.

En cuanto al número y tamaño de las vegetaciones, en tres $(11,11 \%)$ no se encontró vegetación visible o no pudo calcularse el tamaño mediante ecocardiografía; asimismo, $14(51,85 \%)$ presentaron una sola vegetación y en 8 (32\%), más de una vegetación.
Tabla 1. Localización de vegetaciones.

\begin{tabular}{lcc}
\hline Localización & n & \% \\
\hline Válvula mitral & 17 & 62,96 \\
Válvula aórtica & 13 & 48,15 \\
$\begin{array}{l}\text { Otra localización* } \\
\text { *Válvula pulmonar, Válvula tricúspide, Septum } \\
\text { interventricular, Comunicación IV }\end{array}$ & \\
\end{tabular}

Las indicaciones quirúrgicas encontradas fueron riesgo de embolismo, insuficiencia cardiaca refractaria a tratamiento médico e infección no controlada (Tabla 2). En $21(77,78 \%)$ pacientes tuvieron indicación 
Tabla 2. Indicación quirúrgica.

\begin{tabular}{lcc}
\hline Indicación quirúrgica & $\mathbf{n}$ & $\mathbf{\%}$ \\
\hline Riesgo de embolismo & 20 & 74,07 \\
Insuficiencia cardiaca & 19 & 70,37 \\
Infección incontrolable & 14 & 51,85 \\
\hline
\end{tabular}

de cirugía de urgencia y seis $(22,22 \%)$, cirugía de emergencia. No hubo pacientes con indicación de cirugía electiva.

Catorce $(73,68 \%)$ pacientes con insuficiencia cardiaca refractaria a tratamiento médico tuvieron indicación de cirugía de urgencia, el 31,58\% presentó hipertensión pulmonar y en el $68,42 \%$ no se pudo evaluar la presión sistólica pulmonar. De los 20 pacientes con riesgo de embolismo, 65\% tuvo indicación de cirugía de urgencia, debido a la presencia de vegetaciones $\geq 10 \mathrm{~mm}$ con algún evento cardioembólico (20\%) o vegetaciones $\geq 15 \mathrm{~mm}$ (75\%). Por otro lado, $8(57,14 \%)$ pacientes con infección no controlada tuvieron indicación de cirugía de urgencia, de ellos en el 14,29\% se aisló un microorganismo resistente y en $85,71 \%$ hubo persistencia de fiebre por más de 7 días luego de iniciar el tratamiento antibiótico.

En cuanto al tipo de cirugía indicada, 26 (96,3\%) tenían indicación sólo de cambio valvular, uno $(3,70 \%)$ sólo de reparación valvular y uno $(3,70 \%)$ de ambos tipos de cirugía. Sólo siete $(25,93 \%)$ fueron intervenidos quirúrgicamente, de ellos a seis se les realizó cirugía de cambio valvular y a un paciente cirugía de reparación valvular.

Veinte $(70,07 \%)$ pacientes, a pesar de tener indicación de tratamiento quirúrgico, no fueron intervenidos, nueve $(33,33 \%)$ pacientes fallecieron durante el tratamiento médico, ocho (29,63\%) pacientes presentaron complicaciones pre-quirúrgicas (Gráfico 3).

Siete pacientes fueron intervenidos quirúrgicamente. Las complicaciones post-operatorias fueron: coagulopatía en dos pacientes, sangrado torácico en tres, y en 1/7 pacientes, extrasístoles ventriculares, émbolo pulmonar, shock cardiogénico y muerte, respectivamente. El puntaje del Euroscore II como predictor de morbi-mortalidad postquirúrgica resultó en dos de ellos de alto riesgo quirúrgico (Tabla $3)$.

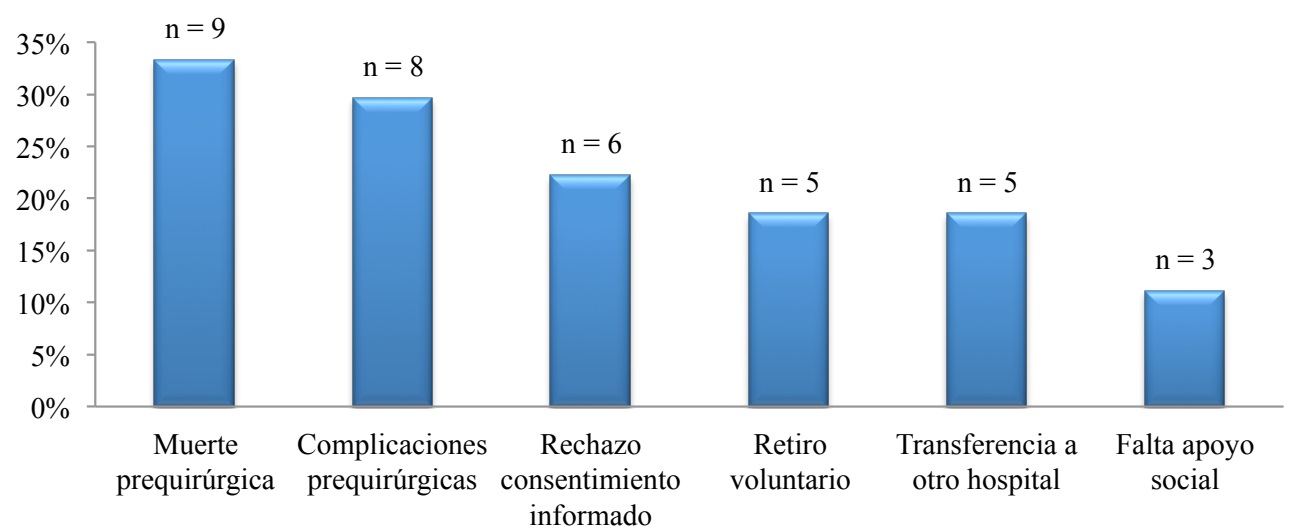

Gráfico 3. Motivos por los cuales no fueron intervenidos quirúrgicamente.

Tabla 3. Puntaje del EuroSCORE II en pacientes con alto riesgo pre-quirúrgico

\begin{tabular}{lc}
\hline EuroSCORE II $(\%)$ & Morbi-mortalidad postquirúrgica \\
\hline 35,47 & Sangrado torácico, IRA \\
23,03 & Coagulopatía, shock cardiogénico y muerte \\
15,65 & Sangrado torácico, coagulopatía, embolismo pulmonar \\
\hline
\end{tabular}




\section{DISCUSIÓN}

Obtuvimos una población bastante similar a la que se describe en otros estudios nacionales e internacionales $(2,5,6,7,8)$, tomando en cuenta la edad, el género, principales factores predisponentes y agentes etiológicos. Sin embargo, contrario a lo que se describe, se encontró un bajo porcentaje de pacientes con uso de drogas endovenosas, lo que podría explicarse debido a la baja incidencia de uso de dichas sustancias en nuestro medio (7).

A pesar de que nuestros pacientes reciben tratamiento antibiótico empírico, se encontró similitud entre los antibióticos más utilizados en nuestro estudio y lo recomendado por las guías americanas y europeas de manejo de endocarditis infecciosa (EI) $(2,4)$.Ninguno de nuestros pacientes recibió profilaxis antibiótica, y según lo estipulado por las guías, sólo uno de nuestros pacientes debió recibirla debido a procedimiento dental (4).

Las guías europeas $(2,4)$ toman en cuenta tres principales indicaciones quirúrgicas, insuficiencia cardiaca refractaria a tratamiento médico, infección severa persistente y prevención de eventos embólicos. La guía americana (3), añade además, complicaciones cerebrovasculares, microorganismos difíciles y endocarditis en válvulas protésicas, los cuales no encontramos en nuestros pacientes.

La indicación más frecuente fue el riesgo de embolismo, contario a lo descrito en la literatura europea y americana, que suele encontrarse en tercer lugar $(3,4)$. Existen varios factores asociados a esta indicación, incluyendo el tamaño y la movilidad de las vegetaciones, la localización de la vegetación en la válvula mitral, el tamaño de la vegetación bajo antibióticoterapia y endocarditis multivalvular (4). Todos los pacientes con indicación de cirugía de urgencia presentaron vegetaciones de tamaño mayor a $10 \mathrm{~mm}$ acompañados de eventos cardioembólicos o mayor de $15 \mathrm{~mm}$., tal como recomiendan las Guías Internacionales $(2,4)$.

La insuficiencia cardiaca refractaria a tratamiento antibiótico es la indicación quirúrgica más frecuente según la literatura $(3,4)$, sin embargo, en nuestro estudio lo encontramos en segundo lugar. La guía europea describe que la insuficiencia cardiaca aparece en mayor número cuando se encuentra afectada la válvula aortica $(4,9)$, contrario a nuestro estudio donde se halló mayor compromiso de la válvula mitral. Esto podría deberse a una mayor incidencia de enfermedad reumática en nuestro medio, ya que, según la Oganización Panamerciana de la Salud (10), el Perú representa un país en transición epidemiológica, donde la población se ve afectada tanto por enfermedades infecciosas, como por enfermedades crónicas no transmisibles. Se menciona (4) que la insuficiencia cardiaca con regurgitación aortica o mitral severa con edema agudo de pulmón y shock cardiogénico es una indicación de cirugía de emergencia. De no ocurrir estos eventos, pero al presentar hipertensión pulmonar, es indicación de cirugía de urgencia. En nuestro estudio, la mayoría tuvieron indicación de cirugía de urgencia, a pesar de no contar con medida de la presión sistólica pulmonar en todos los casos.

La infección no controlada abarca la persistencia de fiebre o hemocultivos positivos después de siete días de tratamiento apropiado, infección por microorganismos resistentes, y extensión perivalvular (4). Esta suele ser la segunda indicación más frecuente a nivel internacional (4), en nuestro estudio ocupó el tercer lugar. El hemocultivo fue positivo en casi la mitad de nuestros pacientes, mientras que en la literatura internacional es de casi $90 \%(2,7)$. Esto podría deberse a debilidades de nuestro laboratorio en la toma de las muestras o en la calidad de los medios de cultivo, pero también a la automedicación en muchos de los pacientes en nuestro medio o haber recibido terapia antibiótica antes de la hospitalización. A pesar de esto, encontramos microorganismos difíciles de tratar como Staphylococcus resistentes como $S$. aureus meticilino resistente, E. coli productores de $\beta$-lactamasas de espectro extendido o Pseudomona multidrogo resistente. Tal y como recomienda la literatura, la mayoría de éstos pacientes tuvo indicación de cirugía de urgencia (4).

Los motivos más frecuentes en los pacientes que no fueron intervenidos, pese a tener indicación quirúrgica, fueron diversas complicaciones que en algunos casos llevaron a la muerte del paciente antes del acto operatorio. Es conocido que la intervención quirúrgica temprana en pacientes con indicación, disminuye la morbi-mortalidad $(3,4,11)$. En nuestro medio existen muchas deficiencias, pues a pesar de tratarse de un establecimiento de Salud nivel III-1 con la especialidad de Cirugía Cardiovascular, no cuenta con un equipo de emergencia para tratar estos casos, y el turno operatorio es solo una vez por semana, lo cual limita el acceso a sala de operaciones. 
En los pacientes que fueron intervenidos quirúrgicamente se cumplió con lo establecido en las guías de manejo europeas (4) con relación al tipo de cirugía. En ellas se recomienda la cirugía de reparación valvular en la mayoría de casos, excepto en los casos donde la lesión valvular pone en peligro la vida del paciente, y en los casos de lesión aórtica, donde lo recomendable es el cambio valvular debido a la complejidad de la reparación y al menos tiempo de uso de circulación extracorpórea $(4,12)$.

Sin pretender una validación del Euroscore II en nuestro medio, se puede evidenciar su utilidad como predictor de morbi- mortalidad postquirúrgica en nuestro estudio.

A pesar de haber encontrado ciertas diferencias en nuestro estudio, el manejo médico y quirúrgico de la endocarditis infecciosa en el Hospital Nacional Cayetano Heredia sigue las recomendaciones de las últimas guías internacionales. Consideramos que las diferencias encontradas podrían deberse al tamaño de nuestro estudio, resaltando así la importancia de realizar estudios mayores y multicéntricos en nuestro medio. Es importante además considerar el gran número de pacientes en los cuales no se llega a realizar la cirugía cardíaca y las razones por las cuales sucede, para así optimizar el manejo de nuestros pacientes.

A pesar de las limitaciones encontradas en nuestro estudio, sobre todo el número reducido de casos, la alta mortalidad y el difícil acceso a las historias clínicas de los pacientes fallecidos; se puede concluir que el manejo de estos pacientes sigue las pautas internacionales, siendo el riesgo de embolismo la indicación quirúrgica más frecuente. Además, la mayoría de los pacientes sometidos a cirugía tuvieron resultados favorables, lo que nos dice de la importancia del manejo quirúrgico en esta patología. Finalmente, es importante considerar el gran número de pacientes que, a pesar de contar con indicación quirúrgica, no logran operarse. Esto nos hace pensar en posibles cambios en nuestro sistema de salud para hacer más accesible el tratamiento quirúrgico a la población.

\section{Declaración de financiamiento y conflicto de intereses:}

El estudio ha sido financiado en su totalidad por los autores. No se han recibido donaciones ni otro tipo de apoyo económico. No existe conflicto de intereses económico, institucional, laboral o personal, de parte de alguno de los autores.

\section{Contribución de autoría:}

$\mathrm{CS}, \mathrm{MP}, \mathrm{AV}$ y EC contribuyeron de manera equitativa con la recolección de información, análisis y redacción del artículo.

\section{Correspondencia:}

Andrea Valle

Las Gaviotas 190- Lima 34

Correo electrónico: andrea.valle@upch.pe

\section{REFERENCIAS BIBLIOGRÁFICAS}

1. Head SJ, Mokhles MM, Osnabrugge R, Bogers A, Kappetein AP. Surgery in current therapy for infective endocarditis. Vascular Health and Risk Management. 2011; 7: 255-263.

2. Habib G. Management of infective endocarditis. Heart. 2006; 92: 124-130.

3. Yamaguchi H, Eishi K. Surgical treatment of active infective mitral valve endocarditis. Ann Thorac Cardiovasc Surg. 2007; 13(3):150-5.

4. Prendergast BD, Tornos P. Surgery for Infective Endocarditis: Who and When? Circulation. 2010; 121: 1141-1152.

5. Lalani T, Cabell CH, Benjamin DK, et al. Analysis of the impact of early surgery on in-hospital mortality of native valve endocarditis: Use of propensity score and instrumental variable methods to adjust for treatment-selection Bias. Circulation. 2010; 121: 1005- 1013.

6. Habib G, Hoen B, Tornos P, et al. Guidelines of the prevention, diagnosis and treatment of infective endocarditis (new version 2009). European Heart Journal. 2009; 30: 2369-2413.

7. Prendergast $\mathrm{BD}$. The changing face of infective endocarditis. Heart. 2006; 92: 879- 885.

8. Westphal N, Plicht B, Naber C. Infective endocarditisprophylaxis, diagnostic criteria, and treatment. Deutsches Ärzteblatt International. 2009; 106 (2829): 481- 490.

9. Tornos P, Iung B, Permanyer-Miralda G, et al. Infective endocarditis in Europe: lessons from the Euro heart survey. Heart. 2005; 91: 571-575.

10. Organización Panamericana de la Salud, Organización Mundial de la Salud. Salud en las Américas. Washington: Organización Panamericana de la Salud; 2012.

11. Hermida A, López R, Rodríguez M, Lado F. Estudio retrospectivo de la endocarditis infecciosa en diferentes grupos de riesgo. Revista Médica de Chile. 2007; 135: 11- 16.

12. Casabé H, Deschle H. Consenso de endocarditis infecciosa. Rev Argent Cardiol. 2002; 70(S5): 1-63. 
13. Romaní F, Cuadra J, Atencia F, Vargas F, Canelo C. Endocarditis infecciosa: análisis retrospectivo en el Hospital Nacional Arzobispo Loayza, 20022007. Revista Peruana de Epidemiología. 2009; 13 (2):1-7.

14. Haider K, Pinsky MR. Early surgery for native valve infective endocarditis. Critical Care. 2013; 17: 304.

15. Delahaye F, Wong J, Mills PG. Infective endocarditis: a comparison of international guidelines. Heart. 2007; 93: 524- 527.
16. O'Gara P. Infective endocarditis 2006: Indications for surgery. Transactions of the American Clinical and Climatological Association. 2007; 118:187-98.

17. Mylonakis E, Calderwood SB. Infective endocarditis in adults. N Engl J Med. 2001; 345 (18): 1318- 1330.

Recibido: 20/03/2014

Aceptado: 08/07/2014 